Provided for non-commercial research and education use. Not for reproduction, distribution or commercial use.

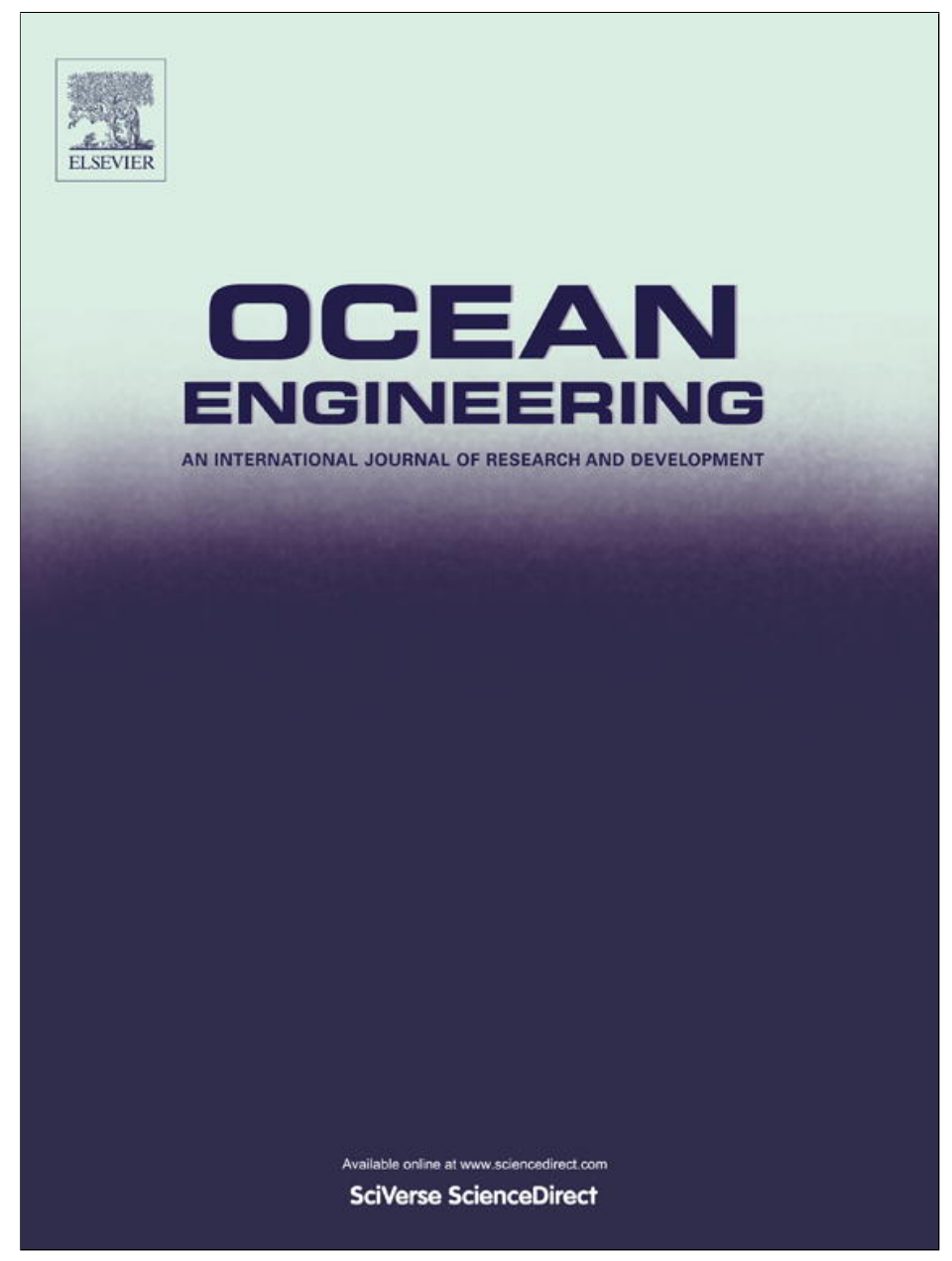

This article appeared in a journal published by Elsevier. The attached copy is furnished to the author for internal non-commercial research and education use, including for instruction at the authors institution and sharing with colleagues.

Other uses, including reproduction and distribution, or selling or licensing copies, or posting to personal, institutional or third party websites are prohibited.

In most cases authors are permitted to post their version of the article (e.g. in Word or Tex form) to their personal website or institutional repository. Authors requiring further information regarding Elsevier's archiving and manuscript policies are encouraged to visit:

http://www.elsevier.com/authorsrights 


\title{
A geometric tool for the analysis of position and force constraints in wave energy converters
}

\author{
Giorgio Bacelli, John V. Ringwood* \\ Centre for Ocean Energy Research, National University of Ireland Maynooth, Co. Kildare, Ireland
}

\section{A R T I C L E I N F O}

\section{Article history:}

Received 1 November 2012

Accepted 18 March 2013

\section{Keywords:}

Wave energy

Power take-off

Constraints

Discretization

\begin{abstract}
A B S T R A C T
Most wave energy devices are subject to finite constraints on both the power take-off (PTO) stroke length and the maximum force that the PTO can tolerate. It is also often the case that greater stroke lengths can reduce the maximum force in the PTO and vice versa. Ultimately, some informed choice of PTO constraints must be made in order to ensure that PTO constraints are not violated and that the trade-off between position and force constraints is made in such as way that maximum energy is captured by the converter. This paper presents a tool to allow device developers to check the satisfaction of constraints for a given hydrodynamic model and set of sea conditions and, where constraints are not satisfied, shows how to relax the constraints to maximize energy capture. The tool is algebraic, requiring no simulation and the results are presented through intuitive geometrical constructs. Sample application results are presented for single- and two-body wave energy systems.
\end{abstract}

(c) 2013 Elsevier Ltd. All rights reserved.

\section{Introduction}

Wave energy conversion technology is at a relatively early stage of maturity, with few commercial devices in the water. However, even at this stage, it is largely accepted that most devices will have two modes of operation. The 'normal' mode of operation is that where power is converted from the waves into a useable form (water pressure, electricity, etc.) and a further 'survivability' mode, which the device will need to enter when the wave excitation forces become too great for the constraints of the normal mode to bear. How each device survives is not the focus of this paper and, indeed, there have been a number of methods proposed, many of which are particular to specific device types. Rather, we concern ourselves with the interplay between the wave excitation force and the physical constraints of the wave energy device, namely the force and position constraints and will assume that, where system constraints cannot be observed, the wave energy device must enter survival mode. We note that there may also be velocity constraints related to limitations of rate of movement of various system components. While velocity constraints can also be included in our formulation, we focus on the main constraints of force and position, for brevity.

The suggested interplay between the force and position (amplitude) constraints, and the wave excitation force, is illustrated by Fig. 1. A situation which satisfies the constraints for a given wave excitation force, $F_{e}$, is indicated by an intersection of the force and position sets. In general, larger position and force constraints are

\footnotetext{
* Corresponding author. Tel.: +35317084766.

E-mail address: john.ringwood@eeng.nuim.ie (J.V. Ringwood).
}

indicated by circles of greater radii, while larger excitation forces are represented by a greater distance between circle centers. Ideally, we would like to identify the functions $f_{1}(), f_{2}()$ and $f_{3}()$ so that the construct in Fig. 1 can be generated for a specific wave energy device. In addition, it would be beneficial if some indication of the energy landscape could be provided so that, where constraints are not immediately satisfied, some guidance is available to allow captured energy to be maximized by virtue of a constraint relaxation.

The feasibility problem, as identified in the preceding paragraph, has, to date, not been addressed. A number of researchers address the issue of position constraints and design a controller which actuates PTO force in order to satisfy constraints, usually focussing position constraints alone. However, such studies presuppose a particular control design method. For example, amplitude constraints, based on a frequency-domain approach, are addressed in Evans (1981), Pizer (1993), and Falnes (2000), while a number of authors have utilized the model predictive control (MPC) framework to maximise energy capture subject to constraints; for example Cretel et al. (2011). The work reported in Hals et al. (2011) is interesting in that it uses MPC to solve for the optimal velocity profile of the wave energy device, subject to amplitude constraints, while then examining the PTO force required to achieve this velocity profile, where the PTO force is also subject to constraints.

Our development is based on the discretization of the equations of motion of the wave energy converters (WECs) by means of the approximation of the forces and of the velocities with a linear combination of basis functions. A special case is considered, in which truncated Fourier series are used for the approximation. 
This case is particularly interesting because the results are in harmony with the frequency domain theory of wave energy conversion, as in Falnes (2002). The discretization was initially motivated by the study of an optimal controller for the maximization of the energy converted by the device, subject to constraints. For brevity, only the theoretical details crucial to the development of the constraint framework are described here. The full details of the underlying control framework are given in Bacelli et al. (2011).

While it is (theoretically) possible to determine the satisfaction of system constraints for a given wave climate via extensive simulation, such an approach would be both time consuming and may not capture the complete set of circumstances. In contrast, the constraint analysis tool developed in this paper is analytical, requiring a hydrodynamic model of the wave energy device of interest together with a specification of the force and position constraints and the excitation force in order to give an immediate conclusion regarding the satisfaction of the constraints. Furthermore, the graphical nature of the answer provides some insight into the interplay between $c_{f}, c_{p}$ and $F_{e}$, placed against the background of absorbed energy and therefore provides the basis for a design tool.

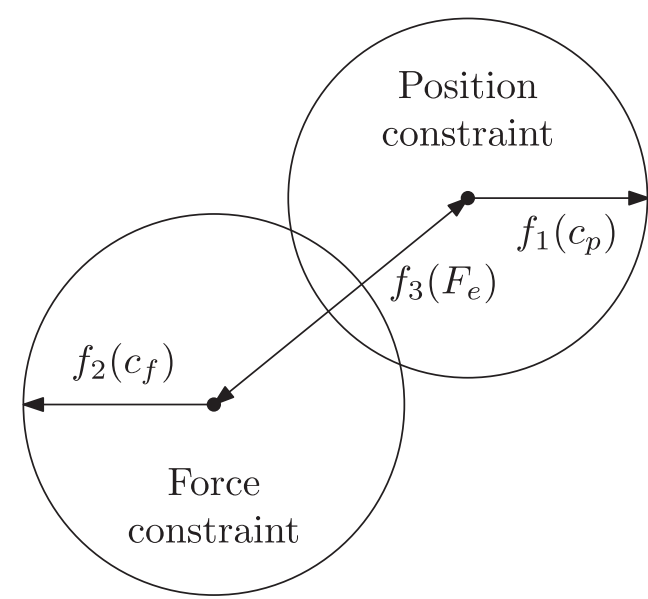

Fig. 1. Illustration of the interplay between excitation force, $F_{e}$, position constraint, $c_{p}$ and force constraint, $c_{f}$.

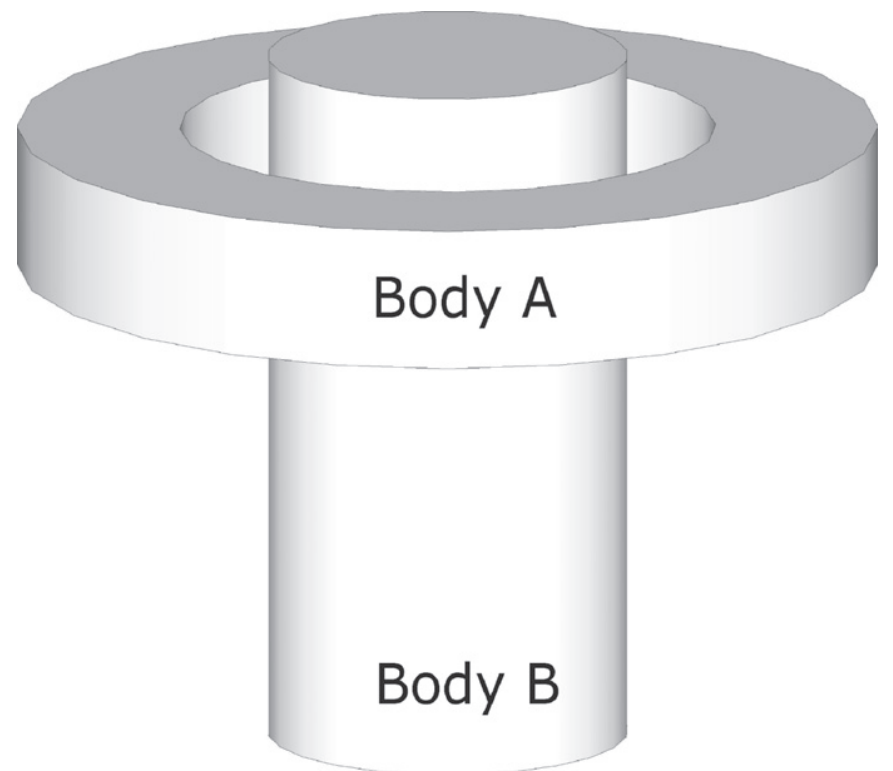

Fig. 2. Self-reacting point absorber.
In the paper we present the theoretical development for a multi (two)-body device, where single body analysis can be achieved as a special case. The paper proceeds as follows: Section 2 presents the mathematical model of a two-body self-reacting point absorber and the discretization performed by means of the Galerkin method. The force and oscillation amplitude constraints are defined in Section 3; the procedure for approximating the constraints by means of the 2-norm is also presented, in conjunction with the geometrical interpretation of the approximated constraints. In Section 4, the case of a single-body point absorber is considered as a special case of the two-body device, while sample results for both single-body and two-body WECs are described in Section 5.

\section{Self-reacting point absorber model}

The general form of device considered is a two-body self-reacting point absorber restricted to heave motion only, as depicted in Fig. 2, and described by the frequency domain model (Falnes, 1999)

$\left\{\begin{array}{l}\left(i \omega m^{A}+B^{A}+\frac{S^{A}}{i \omega}\right) V^{A}=F_{e}^{A}+F_{r}^{A}-F_{p t o} \\ \left(i \omega m^{B}+B^{B}+\frac{S^{B}}{i \omega}\right) V^{B}=F_{e}^{B}+F_{r}^{B}+F_{p t o}\end{array}\right.$

where $V^{A}$ and $V^{B}$ are the vertical velocities of body $A$ and body $B$, respectively. The radiation force $F_{r}$ is

$\left[\begin{array}{c}F_{r}^{A} \\ F_{r}^{B}\end{array}\right]=-Z\left[\begin{array}{l}V^{A} \\ V^{B}\end{array}\right]$ with $Z=\left[\begin{array}{ll}Z^{A A} & Z^{A B} \\ Z^{B A} & Z^{B B}\end{array}\right]$

where $Z=Z(\omega)$ is the radiation impedance matrix.

$F_{e}{ }^{B}$ and $F_{e}{ }^{B}$ denote the excitation forces, $m^{A}$ and $m^{B}$ are the masses, $S^{A}$ and $S^{B}$ the hydrodynamic stiffness coefficients, and $B^{A}$ and $B^{B}$ are damping coefficients used to model linear losses due to viscous effects and frictions of mechanical components. The damping and stiffness coefficients can also embed the terms describing the linear model for mooring loads; for example, considering body A, this can be done by redefining both $S^{A}$ and $B^{A}$ as $S^{A}=S_{M}^{A}+S_{H}^{A}$ and $B^{A}=B_{M}^{A}+B_{H}^{A}$, where the subscript $M$ denotes terms due to the mooring loads and the subscript $H$ refer to the hydrostatic $\left(S_{H}^{A}\right)$, viscous and other linear mechanical dissipative effects $\left(B_{H}^{A}\right)$. The PTO applies a force $F_{p t o}$ on both bodies with the same magnitude and opposite direction. The time domain formulation of the model in (1) is

$\left\{\begin{array}{l}m^{A} \dot{v}^{A}(t)+B^{A} v^{A}(t)+S^{A} u^{A}(t)-f_{r}^{A}(t)=f_{e}^{A}(t)-f_{p t o}(t) \\ m^{B} \dot{v}^{B}(t)+B^{B} v^{B}(t)+S^{B} u^{B}(t)-f_{r}^{B}(t)=f_{e}^{B}(t)+f_{p t o}(t)\end{array}\right.$

where $u^{A}(t)$ and $u^{B}(t)$ are the vertical positions of the two bodies, and $\dot{v}^{A}(t)$ and $\dot{v}^{B}(t)$ their vertical accelerations. The forces and velocities, denoted with lowercase letters in (2), are the inverse Fourier transform of the corresponding uppercase variables in (1). The radiation forces are

$f_{r}^{A}(t)=-m_{\infty}^{A A} \dot{v}^{A}(t)-k^{A A}(t) * v^{A}(t)-m_{\infty}^{A B} \dot{v}^{B}(t)-k^{A B}(t) * v^{B}(t)$

$f_{r}^{B}(t)=-m_{\infty}^{B B} \dot{v}^{B}(t)-k^{B B}(t) * v^{B}(t)-m_{\infty}^{B A} \dot{v}^{A}(t)-k^{B A}(t) * v^{A}(t)$

where the symbol $*$ denotes the convolution operator and the parameters $m_{\infty}^{i j}$ and $k^{i j}(t)$, with $i, j \in\{A, B\}$, are related to the elements of the radiation impedance matrix $Z$ through the expressions (Ogilvie, 1964)

$$
\begin{aligned}
& m^{i j}(\omega)=\operatorname{Im}\left\{Z^{i j}(\omega)\right\}=m_{\infty}^{i j}-\frac{1}{\omega} \int_{0}^{\infty} k^{i j}(t) \sin (\omega t) d t, \\
& R^{i j}(\omega)=\operatorname{Re}\left\{Z^{i j}(\omega)\right\}=\int_{0}^{\infty} k^{i j}(t) \cos (\omega t) d t,
\end{aligned}
$$


$m_{\infty}^{i j}=\lim _{\omega \rightarrow \infty} \operatorname{Im}\left\{Z^{i j}(\omega)\right\}$.

The energy absorbed in the interval $[0, T]$, neglecting losses, corresponds to the work performed by the PTO, that is

$J(T)=\int_{0}^{T} f_{p t o}(t)\left(v^{A}(t)-v^{B}(t)\right) d t$.

\subsection{Discretization}

The PTO force is assumed to be such that $f_{p t o}(t) \in L^{2}([0, T])$, where $L^{2}([0, T])$ is the Hilbert space of square integrable functions in the interval $[0, T]$. The velocities and the accelerations are also assumed to be square integrable, that is $v^{i}(t) \in H^{1}([0, T])$ where $H^{1}([0, T])$ is the Sobolev space of square integrable functions in $[0, T]$, the derivatives of which are also square integrable. The PTO force and the velocities are then approximated as a linear combination of basis functions in a finite dimensional subspace of the space $L^{2}([0, T])$

$v^{i}(t) \approx \hat{v}^{i}(t)=\sum_{n=1}^{N} x_{n}^{i} \phi_{n}(t), \quad i \in\{A, B\}$

$f_{p t o}(t) \approx \hat{f}_{p t o}(t)=\sum_{n=1}^{N^{P}} p_{n} \phi_{n}^{P}(t)$

where $\left\{\phi_{1}(t), \ldots, \phi_{N}(t)\right\}$ is a basis for the finite dimensional subspace $S^{V} \subset H^{1}([0, T])$ and $\left\{\phi_{1}^{P}(t), \ldots, \phi_{N^{p}}^{P}(t)\right\}$ is a basis for the finite dimensional subspace $S^{P} \subset L^{2}([0, T])$. For any given set of coefficients describing the PTO force $\left\{p_{1}, \ldots, p_{N^{p}}\right\}$ and excitation forces $f_{e}^{A}$ and $f_{e}^{B}$, the components of the velocities are calculated by applying the Galerkin method (Boyd, 2001)

$\left\{\begin{array}{l}\left\langle\hat{r}^{A}(t), \phi_{n}\right\rangle=0 \\ \left\langle\hat{r}^{B}(t), \phi_{n}\right\rangle=0\end{array} \quad \forall n=1, \ldots, N\right.$

where $\langle\cdot, \cdot\rangle$ denotes the inner product defined as

$\langle f, g\rangle=\int_{0}^{T} f(t) g(t) d t$,

and $\hat{r}^{A}(t)$ and $\hat{r}^{B}(t)$ are the equations of motion (2) in the residual form, with the velocities and the forces approximated by using (5) and (6), that is

$\left\{\begin{array}{l}\hat{r}^{A}(t)=m^{A} \dot{\hat{v}}^{A}(t)+B^{A} \hat{v}^{A}(t)+S^{A} \hat{u}^{A}(t)-f_{r}^{A}(t)-f_{e}^{A}(t)+f_{p t o}(t) \\ \hat{r}^{B}(t)=m^{B} \dot{\hat{v}}^{B}(t)+B^{B} \hat{v}^{B}(t)+S^{B} \hat{u}^{B}(t)-f_{r}^{B}(t)-f_{e}^{B}(t)-f_{p t o}(t)\end{array}\right.$

where $\hat{u}^{i}(t)$ are the approximated vertical positions, which are obtained by integrating (5). The Galerkin method is a projection method for the discretization of integral and differential equations; the solution of (7) is the set of the velocity components $x_{n}{ }^{i}$ that minimizes the difference between the velocities obtained by solving the original equations of motion (2) and their approximations (5). In other words, for any given PTO force and excitation forces, the solution of Eq. (7) provides the best approximation of the motion of the WEC in terms of the velocity components.

Given the oscillatory nature of the problem, a truncated Fourier series, also known as a trigonometric polynomial, is an intuitive choice as the basis for $S^{V}$ and $S^{P}$. Furthermore, choosing $w_{0}=2 \pi / T$, the set of functions $\left\{\sin \left(\omega_{0} T\right), \cos \left(\omega_{0} T\right), \ldots, \sin \left(N \omega_{0} T\right), \cos \left(N \omega_{0} T\right)\right\}$ form an orthogonal basis for the spaces $S^{V}$ and $S^{P}$ with the inner product defined in (8). The constant term of the basis is not considered because it is assumed that all the functions have zero mean. In practice, it is assumed that the reference frames of the bodies are chosen such that the origins oscillate around their mean position with respect to the inertial reference frame.
Using a zero mean truncated Fourier series with $N$ frequency components for both the velocities and the PTO force, the dimension of each of the spaces $S^{V}$ and $S^{P}$ is $2 N$, and the resulting approximating functions in (5) and (6) become

$\hat{v}^{A}(t)=\sum_{n=1}^{N} a_{n}^{A} \cos \left(n \omega_{0} t\right)+b_{n}^{A} \sin \left(n \omega_{0} t\right)$

$\hat{v}^{B}(t)=\sum_{n=1}^{N} a_{n}^{B} \cos \left(n \omega_{0} t\right)+b_{n}^{B} \sin \left(n \omega_{0} t\right)$

$\hat{f}_{p t o}(t)=\sum_{n=1}^{N} a_{n}^{P} \cos \left(n \omega_{0} t\right)+b_{n}^{P} \sin \left(n \omega_{0} t\right)$.

For the practical implementation of the method, it is also convenient to approximate the excitation forces by a truncated Fourier series containing $N$ frequency components

$f_{e}^{A}(t) \approx \hat{f}_{e}^{A}(t)=\sum_{n=1}^{N} e_{n}^{A_{c}} \cos \left(n \omega_{0} t\right)+e_{n}^{A_{s}} \sin \left(n \omega_{0} t\right)$

$f_{e}^{B}(t) \approx \hat{f}_{e}^{B}(t)=\sum_{n=1}^{N} e_{n}^{B_{c}} \cos \left(n \omega_{0} t\right)+e_{n}^{B_{s}} \sin \left(n \omega_{0} t\right)$.

The mean value of the excitation forces can be considered to be zero with no loss of generality. In fact, since the excitation force is calculated by the convolution of the wave elevation with the excitation force kernel (Falnes, 1999), the wave elevation can be transformed into a zero mean function by changing the origin of the reference frame, resulting in a zero mean excitation force.

Substituting Eqs. (9)-(13) into (2), the system of (7) becomes the linear system (Bacelli et al., 2011)

$\left[\begin{array}{ll}G^{A A} & G^{A B} \\ G^{B A} & G^{B B}\end{array}\right]\left[\begin{array}{l}X^{A} \\ X^{B}\end{array}\right]=\left[\begin{array}{l}E^{A} \\ E^{B}\end{array}\right]+\left[\begin{array}{c}-I_{2 N} \\ I_{2 N}\end{array}\right] P$

where $I_{2 N}$ is the identity matrix of size $2 N$

$X^{A}=\left[a_{1}^{A}, b_{1}^{A}, a_{2}^{A}, b_{2}^{A}, \ldots, a_{N}^{A}, b_{N}^{A}\right]^{T}$,

$X^{B}=\left[a_{1}^{B}, b_{1}^{B}, a_{2}^{B}, b_{2}^{B}, \ldots, a_{N}^{B}, b_{N}^{B}\right]^{T}$,

$E^{A}=\left[e_{1}^{A_{c}}, e_{1}^{A_{s}}, e_{2}^{A_{c}}, e_{2}^{A_{s}}, \ldots, e_{N}^{A_{c}}, e_{N}^{A_{s}}\right]^{T}$,

$E^{B}=\left[e_{1}^{B_{c}}, e_{1}^{B_{s}}, e_{2}^{B_{c}}, e_{2}^{B_{s}}, \ldots, e_{N}^{B_{c}}, e_{N}^{B_{s}}\right]^{T}$,

$P=\left[a_{1}^{p}, b_{1}^{p}, a_{2}^{p}, b_{2}^{p}, \ldots, a_{N}^{p}, b_{N}^{p}\right]^{T}$,

$G^{i j}=\left[\begin{array}{cccccc}D_{1}^{i j} & M_{1}^{i j} & 0 & \cdots & 0 & 0 \\ -M_{1}^{i j} & D_{1}^{i j} & 0 & \cdots & 0 & 0 \\ 0 & 0 & \ddots & & \vdots & \vdots \\ \vdots & \vdots & & \ddots & 0 & 0 \\ 0 & 0 & \cdots & 0 & D_{N}^{i j} & M_{N}^{i j} \\ 0 & 0 & \cdots & 0 & -M_{N}^{i j} & D_{N}^{i j}\end{array}\right]$,

with $i, j=\{A, B\}$, and

$D_{n}^{i i}=R^{i i}\left(n \omega_{0}\right)+B^{i}$,

$M_{n}^{i i}=n \omega_{0}\left(m^{i}+m^{i i}\left(n \omega_{0}\right)\right)-S^{i} /\left(n \omega_{0}\right)$,

$D_{n}^{i j}=R^{i j}\left(n \omega_{0}\right) \quad$ for $\quad i \neq j$

$M_{n}^{i j}=n \omega_{0} m^{i j}\left(n \omega_{0}\right)$ for $\quad i \neq j$.

The matrix $G_{i j}$ is block diagonal and each block is a 2-by-2 normal matrix of the form

$\left[\begin{array}{cc}a & b \\ -b & a\end{array}\right]$

This particular structure is due to the orthogonality of the Fourier series and it allows the study of the existence of the solution of the 
linear system in (14) by studying the singularity of each of the $N$ 4by-4 matrices

$G_{n}=\left[\begin{array}{cccc}D_{n}^{A A} & M_{n}^{A A} & D_{n}^{A B} & M_{n}^{A B} \\ -M_{n}^{A A} & D_{n}^{A A} & -M_{n}^{A B} & D_{n}^{A B} \\ D_{n}^{B A} & M_{n}^{B A} & D_{n}^{B B} & M_{n}^{B B} \\ -M_{n}^{B A} & D_{n}^{B A} & -M_{n}^{B B} & D_{n}^{B B}\end{array}\right]$.

Each matrix $G_{n}$ corresponds to a frequency $n \omega_{0}$; thus, should the system in (14) be singular, a possible solution might be to perform a different frequency discretization by selecting a different fundamental frequency $\omega_{0}$.

If the solution of the linear system in (14) exists, the amount of energy absorbed by the PTO, evaluated by (4), is

$J(P)=-P^{T} H P+P^{T}\left(Q^{A} E^{A}-Q^{B} E^{B}\right)$

where

$H=S_{G^{B B}}^{-1}+G^{A A^{-1}} G^{A B} S_{G^{A A}}^{-1}+G^{B B^{-1}} G^{B A} S_{G^{B B}}^{-1}+S_{G^{A A}}^{-1}$,

$Q^{A}=S_{G^{B B}}^{-1}+G^{B B^{-1}} G^{B A} S_{G^{B B}}^{-1}$

$Q^{B}=G^{A A^{-1}} G^{A B} S_{G^{A A}}^{-1}+S_{G^{A A}}^{-1}$.

$S_{G^{A A}}$ and $S_{G^{B B}}$ are, respectively, the Schur complements of $G^{A A}$ and $G^{B B}$, and are defined as

$S_{G^{A A}}=G^{B B}-G^{B A} G^{A A^{-1}} G^{A B}$

$S_{G^{B B}}=G^{A A}-G^{A B} G^{B B^{-1}} G^{B A}$.

It can be verified that the symmetric part of the matrix $H$ (i.e. $\left.\left(H+H^{T}\right) / 2\right)$ is positive definite; therefore, the quadratic cost function in (22) is concave and the global maximum of the unconstrained problem is obtained for

$\bar{P}=\left(H+H^{\prime}\right)^{-1}\left(Q^{A} E^{A}-Q^{B} E^{B}\right)$.

\section{Specification and approximation of constraints}

The force constraint is defined as

$\left\|\hat{f}_{\text {pto }}\right\|_{\infty} \leq F_{\text {max }}$,

while the constraint on the relative amplitude is

$\|\Delta \hat{u}\|_{\infty} \leq \Delta U_{\max }$,

where the infinity norm $\|\cdot\|_{\infty}$ is defined in the appendix (Eq. (A.2)) and $\Delta \hat{u}(t)=\hat{u}^{A}(t)-\hat{u}^{B}(t)$, with

$\hat{u}^{A}(t)=u_{0}^{A}+\sum_{j=1}^{N} \frac{b_{n}^{A}}{n \omega_{0}}\left(1-\cos \left(n \omega_{0} t\right)\right)+a_{n}^{A} \sin \left(n \omega_{0} t\right)$,

$\hat{u}^{B}(t)=u_{0}^{B}+\sum_{j=1}^{N} \frac{b_{n}^{B}}{n \omega_{0}}\left(1-\cos \left(n \omega_{0} t\right)\right)+a_{n}^{B} \sin \left(n \omega_{0} t\right)$.

\subsection{Constraint approximation}

While the infinity norm describes how the constraints are actually specified i.e. in terms of absolute limits, the infinity norm is not the easiest to manipulate mathematically and does not easily lead to geometric interpretation. The result in the Appendix allows us rearrange the constraint conditions in terms of the 2-norm, which is more convenient to use.

Using the inequality in (A.4) (see Appendix), it is possible to find sufficient conditions for the satisfaction of the inequalities in (29) and (30) in terms of the 2-norm, (A.1)

$\left\|\hat{f}_{p t o}\right\|_{\infty} \leq \sqrt{\frac{2 N}{T}}\left\|\hat{f}_{p t o}\right\|_{2} \leq F_{\max }$,

$\|\Delta \hat{u}\|_{\infty} \leq \sqrt{\frac{2 N}{T}}\|\Delta \hat{u}\|_{2} \leq \Delta U_{\max }$.

Inequalities (33) and (34) specify the 2-norm scaled by $\sqrt{2 N / T}$ as an upper bound for the infinity norm.

Sufficient conditions for the violation of at least one of the constraints, also in terms of the 2-norm, can be obtained using the inequality in (A.3)

$\left\|\hat{f}_{\text {pto }}\right\|_{\infty} \geq 1 / \sqrt{T}\left\|\hat{f}_{\text {pto }}\right\|_{2} \geq F_{\max }$

$\|\Delta \hat{u}\|_{\infty} \geq 1 / \sqrt{T}\|\Delta \hat{u}\|_{2} \geq \Delta U_{\max }$.

Therefore, the inequalities of (35) and (36) show that the 2-norm scaled by $1 / \sqrt{T}$ provides a lower bound for the infinity norm. Therefore, if the 2 -norm multiplied by $1 / \sqrt{T}$ violates a constraint, then the infinity norm also violates it.

A conceptual figure, showing the relative magnitudes of scaled infinity and 2-norms, for a dummy variable $X_{d}$, is shown in Fig. 3.

\subsection{Constraint calculation}

The 2-norm of the force and amplitude constraints are easily calculated by applying Parseval's theorem, resulting in

$$
\begin{aligned}
\left\|\hat{f}_{p t o}\right\|_{2}^{2} & =\frac{T}{2} \sum_{n=1}^{N}\left(\left(a_{n}^{p}\right)^{2}+\left(b_{n}^{p}\right)^{2}\right)=\frac{T}{2} P^{T} P, \\
\|\Delta \hat{u}\|_{2}^{2} & =\frac{T}{2} \sum_{n=1}^{N} \frac{1}{n \omega_{0}}\left(\left(b_{n}^{A}-b_{n}^{B}\right)^{2}+\left(a_{n}^{A}-a_{n}^{B}\right)^{2}\right) \\
& =\frac{T}{2}\left(X^{A}-X^{B}\right)^{T} W^{2}\left(X^{A}-X^{B}\right),
\end{aligned}
$$

where the amplitude constraint is obtained by setting the initial relative position $\Delta \hat{u}(0)$ as

$\Delta \hat{u}(0)=\sum_{n=1}^{N} \frac{b_{n}^{B}-b_{N}^{A}}{n \omega_{0}}$

to obtain a zero mean valued relative position, and the matrix $W$ is

$W=\left[\begin{array}{ccccc}1 / \omega_{0} & 0 & \cdots & 0 & 0 \\ 0 & 1 / \omega_{0} & \cdots & 0 & 0 \\ \vdots & \vdots & \ddots & \vdots & \vdots \\ 0 & 0 & \cdots & 1 / N \omega_{0} & 0 \\ 0 & 0 & \cdots & 0 & 1 / N \omega_{0}\end{array}\right]$

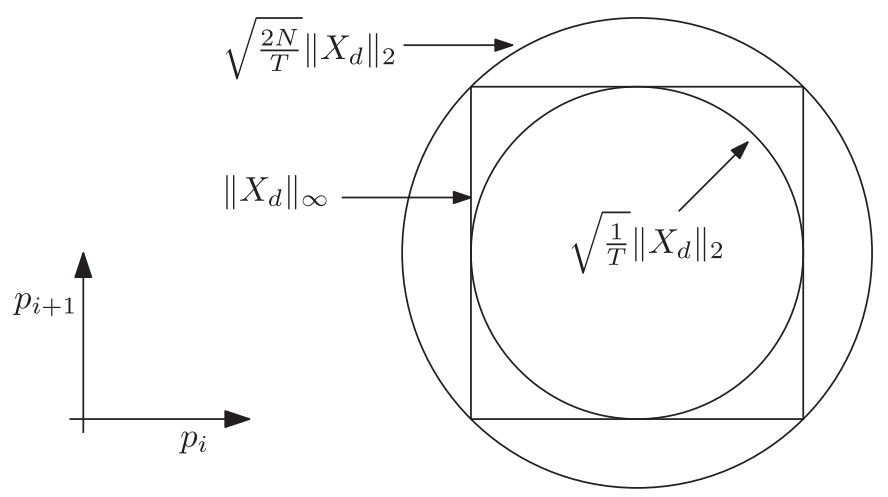

Fig. 3. Conceptual illustration of the relationship between infinity and 2-norms. 
Using Eqs. (23)-(25), the 2-norm of the amplitude constraint in Eq. (38) becomes a function of the vector $P$

$\|\Delta \hat{u}\|_{2}^{2}=\frac{T}{2}\left(P^{T} H^{T} W^{2} H P-2 Q^{T} W^{2} H P+Q^{T} W^{2} Q\right)$.

Substituting Eqs. (37) and (40) into inequalities (33) and (34), the sufficient conditions for satisfaction of the constraints are

$P^{T} P \leq \frac{1}{N} F_{\max }^{2}$

and

$P^{T} H^{T} W^{2} H P-2 Q^{T} W^{2} H P+Q^{T} W^{2} Q \leq \frac{1}{N} \Delta U_{\max }^{2}$,

while the sufficient conditions for violation of the constraints are

$P^{T} P \geq 2 F_{\max }^{2}$

and

$P^{T} H^{T} W^{2} H P-2 Q^{T} W^{2} H P+Q^{T} W^{2} Q \geq 2 \Delta U_{\max }^{2}$.

Inequalities (41)-(44) describe the constraints as a function of the PTO force only, in terms of the vector $P$, for given excitations $E^{A}$ and $E^{B}$, contained in the vector $Q$.

\subsection{Geometrical interpretation}

Defining the sets

$S_{f}\left(R_{f}\right)=\left\{P: P^{T} P \leq R_{f}\right\}$

and

$S_{u}\left(R_{u}\right)=\left\{P: P^{T} H^{T} W^{2} H P-2 Q^{T} W^{2} H P+Q^{T} W^{2} Q \leq R_{u}\right\}$

a sufficient condition for the satisfaction of both the force and the amplitude constraints described by the (29) and (30) is

$S_{f}\left(\frac{1}{N} F_{\text {max }}^{2}\right) \cap S_{u}\left(\frac{1}{N} \Delta U_{\text {max }}^{2}\right) \neq \varnothing$,

that is, if the (2-norm) lower bounds on the force and amplitude constraints intersect, there is a feasible solution for force and amplitude within the constraints. Clearly, from Fig. 3, the condition in (47) is slightly conservative, since the sets corresponding to the infinity norms may intersect, even if those corresponding to the 2-norms do not.

From the upper bound on the infinity norm (Fig. 3) we can also specify a sufficient condition for the violation of at least one of the constraints in (29) or (30) as

$S_{f}\left(2 F_{\text {max }}^{2}\right) \cap S_{u}\left(2 \Delta U_{\text {max }}^{2}\right)=\varnothing$.

The force constraint defined by the set $S_{f}\left(R_{f}\right)$ can be interpreted as the region of the $2 N$-dimensional space $S^{P}$ enclosed by the hypersphere centered at the origin and of radius $\sqrt{R_{f}}$. The amplitude constraint $S_{u}\left(R_{u}\right)$ is the region of the space enclosed by the hyperellipsoid with axes parallel to the elements of the basis of $S^{P}$, because the matrix $H^{T} W^{2} H$ is diagonal and with all positive elements. The center $P_{c}$ of the hyperellipsoid is

$P_{c}=H^{-1} Q=H^{-1}\left(Q^{A} E^{A}-Q^{B} E^{B}\right)$,

while the radii $r_{i}$ are given by

$r_{i}=\sqrt{\frac{R_{u}}{\lambda_{i}}}$

where $\lambda_{i}$ are the eigenvalues of $H^{T} W^{2} H$, that is the diagonal elements.

Eq. (47) states that if the intersection between the hypersphere describing the force constraint and the hyperellipsoid describing the amplitude constraint is not the empty set, then, for the given excitation forces, the device is able to satisfy both the amplitude and the force constraints. Conversely, Eq. (48) states that if the intersection between the two sets is the empty set, then at least one constraint will be violated.

\section{Special case of single body}

A single body heaving buoy is now considered as a special case of the self-reacting point absorber described in Section 2, given by the frequency domain model

$(i \omega m+B+Z(\omega)+S / i \omega) V=F_{e}-F_{p t o}$.

The corresponding time domain model is

$\left(m+m_{\infty}\right) \dot{v}(t)+k(t) * v(t)+B v(t)+S u(t)=f_{e}(t)-f_{p t o}(t)$,

and the energy absorbed by the PTO, neglecting losses, is

$J(T)=\int_{0}^{T} f_{p t o}(t) v(t) d t$.

The energy maximization problem is then discretized by following the same steps performed for the case of a self-reacting device (Section 2.1). That is, the excitation force, the velocity and the PTO force are approximated using truncated Fourier series as in Eqs. (9), (11) and (12); the best approximation is then obtained by applying the Galerkin method for the minimization of the residual $\hat{r}(t)$, which consists in solving

$\left\langle\hat{r}(t), \phi_{n}\right\rangle=0 \quad \forall n=1, \ldots N$,

and results in the linear system

$G X=E-P$.

The matrix $G$ is block diagonal with 2-by-2 blocks of the form of Eq. (20); the elements on the main diagonal are the real parts of the mechanical impedance at the $N$ frequencies $n \omega_{0}$, as defined in Eqs. (16)-(19). If the matrix $G$ is invertible, the converted energy is

$J(P)=P^{T} X=-P^{T} G^{-1} P+P^{T} G^{-1} E$

where the symmetric part of the matrix $G^{-1}$ is positive definite, because the elements on its main diagonal (linear damping plus radiation resistance) are positive. As a consequence, the quadratic function $J(P)$ is concave and the PTO force $\bar{P}$ that maximizes $J(P)$ in $(56)$ is

$\bar{P}=\left(G^{-T}+G^{-1}\right)^{-1} G^{-1} E$.

The 2-norm of the force constraint is defined as in (37), while the 2-norm of the amplitude constraint becomes

$\|\hat{u}\|_{2}^{2}=\frac{T}{2}\left(P^{T} G^{-T} W^{2} G^{-1} P-2 E^{T} G^{-T} W^{2} G^{-1} P+E^{T} G^{-T} W^{2} G^{-1} E\right)$.

Defining the set

$$
\begin{aligned}
S^{\prime}{ }_{u}\left(R_{u}\right)= & \left\{P: P^{T} G^{-T} W^{2} G^{-1} P-2 E^{T} G^{-T} W^{2} G^{-1} P\right. \\
& \left.+E^{T} G^{-T} W^{2} G^{-1} E \leq R_{u}\right\},
\end{aligned}
$$

where the set $S^{\prime}{ }_{u}\left(R_{u}\right)$ describes the region of the space $S^{P}$ enclosed by a hyperellipsoid centered at $P_{c}=E$; the principal axes are parallel to the elements of the basis because the matrix $G^{-T} W^{2} G^{-1}$ is diagonal and the radii are

$r_{i}^{\prime}=\sqrt{\frac{R_{u}}{\lambda_{i}^{\prime}}}$

where $\lambda_{i}^{\prime}$ are the eigenvalues of $G^{-T} W^{2} G^{-1}$.

The case for $N=1$ (i.e. 2-dimensions, $P_{1}$ and $P_{2}$ ) is shown in Fig. 4; the matrix $G^{-1}$ is of the form in Eq. (20), thus $G^{-T} W^{2} G^{-1}$ is diagonal with two coincident eigenvalues $\lambda^{\prime}$, and the set $S^{\prime}{ }_{u}\left(U_{\max }^{2}\right)$ is a disc centered at $P_{c}=E$ with radius $r^{\prime}=U_{\max } / \sqrt{\lambda^{\prime}}$. Considering that the intersection between two disks is non-empty if the sum of the radii is larger that the distance between the centers, it follows 


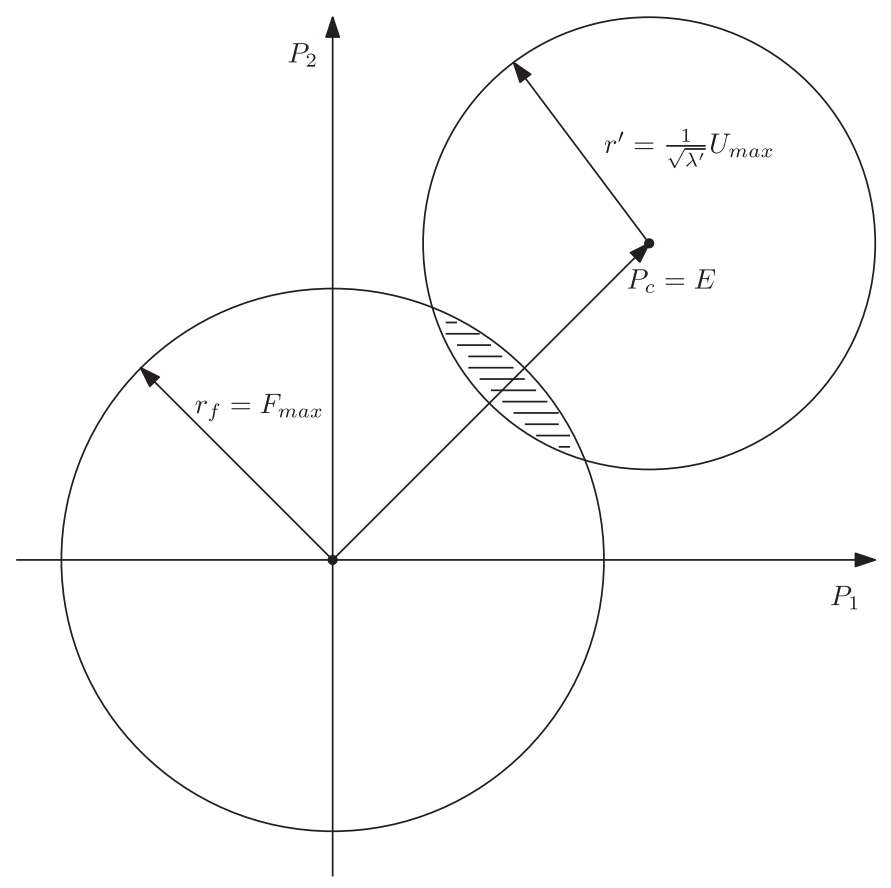

Fig. 4. Example for one-body device, with $N=1$. The axes $P_{1}$ and $P_{2}$ are the components of the vector $P$ describing the PTO force, that is $P=\left[P_{1} P_{2}\right]^{T}$.

that the sufficient condition in Eq. (47) can be simplified to

$F_{\max }+\frac{U_{\max }}{\sqrt{\lambda^{\prime}}} \geq\|E\|_{2}$.

Fig. 4 has a useful physical interpretation in that the larger bounds on amplitude and force result in larger disks for $S^{\prime}{ }_{u}\left(R_{u}\right)$ and $S_{f}\left(R_{f}\right)$ respectively, with larger constraints increasing the likelihood of an intersection between $S_{u}^{\prime}\left(R_{u}\right)$ and $S_{f}\left(R_{f}\right)$. The other important variable affecting intersection is the distance between the disk centers which, for the one-body case, is simply the excitation force experienced by the body. Therefore, for more violent sea states, there is a lower likelihood of simultaneously satisfying position and amplitude constraints, as expected.

Eq. (48), for $N=1$, can also be simplified to

$\sqrt{2}\left(F_{\max }+\frac{U_{\max }}{\sqrt{\lambda^{\prime}}}\right) \leq\|E\|_{2}$.

The geometrical construct in Fig. 4 is now of the form of the originally desired specification, shown in Fig. 1, where we can identify the functions $f_{1}\left(0, f_{2} O\right.$ and $f_{3}()$. A broad conclusion, from Fig. 4 , is that if the circle centered at $P_{c}$ contains the origin, no PTO force is required to limit the motion in order to satisfy the amplitude constraint i.e. the wave excitation force can always be contained within the amplitude range of the device.

\section{Sample results}

Initially, in Section 5.1, we will take a single body device and perform a constraint analysis for a monochromatic sea. This will allow us to develop some insight into the use of the geometrical tool and to consider it from a design perspective. While monochromatic analysis might seem restrictive, appropriate choice of the frequency, such as the resonant frequency of the device, or the peak energy frequency of the incident sea, could be sufficient to determine the peak loading condition. However, a polychromatic approach, for the single body case, is also considered in Section 5.1.2. Finally, we show results for a multi-body device with a polychromatic sea spectrum, in Section 5.2. In all the cases considered, the hydrodynamic parameters were calculated using the WAMIT software from MIT.

\subsection{Single body}

Here, we consider a vertical cylinder with diameter $10 \mathrm{~m}$, draft $25 \mathrm{~m}$ and mass of $4.9 \times 10^{5} \mathrm{~kg}$. The incident sea is of $3 \mathrm{~m}$ amplitude, with a wave period of $11.4 \mathrm{~s}$. PTO amplitude and force constraints are set at $3 \mathrm{~m}$ and $200 \mathrm{kN}$ respectively. The body has a resonant frequency of $0.63 \mathrm{rads} / \mathrm{s}(\equiv 9.93 \mathrm{~s})$.

\subsubsection{Single frequency analysis}

Fig. 5 shows the situation, with respect to the sufficient conditions for satisfaction of the constraints in (61), for a frequency of $0.7 \mathrm{rads} / \mathrm{s}$ (corresponding to a period of $9 \mathrm{~s}$ ). Clearly, there is an intersection between the circles corresponding to the amplitude and force constraints, and the optimal PTO force (for this constrained situation) is expressed by $\bar{P}_{\text {constr }}$. Fig. 5 also displays the optimal unconstrained PTO force, $\bar{P}$, and the contours corresponding to lines of constant energy. Therefore, it can be seen that, should we wish to relax one or other constraint in order to increase captured power, increasing the force constraint would cause the $\bar{P}_{\text {constr }}$ point to move across the energy contours towards $\bar{P}$, while increasing the amplitude constraint yields very little extra energy.

\subsubsection{Multiple frequency analysis}

While the analysis at a single frequency of Section 5.1.1 has some useful design features, real seas contain multiple frequency components and designers need to ensure that physical constraints are satisfied for all active frequencies. In Fig. 6, we consider the same heaving cylinder in a sea state corresponding to a Bretschneider wave spectrum with parameters $H_{m o}=6.5 \mathrm{~m}$ and $T_{p}=10 \mathrm{~s}$. In essence, the plane of Fig. 5 is extended to 3 dimensions, with the addition of a frequency axis.

OHowever, this increase in dimension of the figure brings a corresponding reduction in the clarity of interpretation but we can effectively project the figure information onto a 2 dimensional plane. Fig. 7 shows this projection, which essentially utilises Eqs. (61) and (62) for the frequency range of interest i.e. $\|E\|_{2} /\left(F_{\max }+\right.$ $\left.U_{\max } / \sqrt{\lambda^{\prime}}\right)$ is plotted with respect to both 1 and $\sqrt{2}$. The shaded area of Fig. 7 is an area in which constraints are not guaranteed to be satisfied, but are also not guaranteed to be violated; in essence, the true infinity norms corresponding to $\Delta \hat{u}$ and $\hat{f}_{\text {pto }}$ lie somewhere in the shaded region.

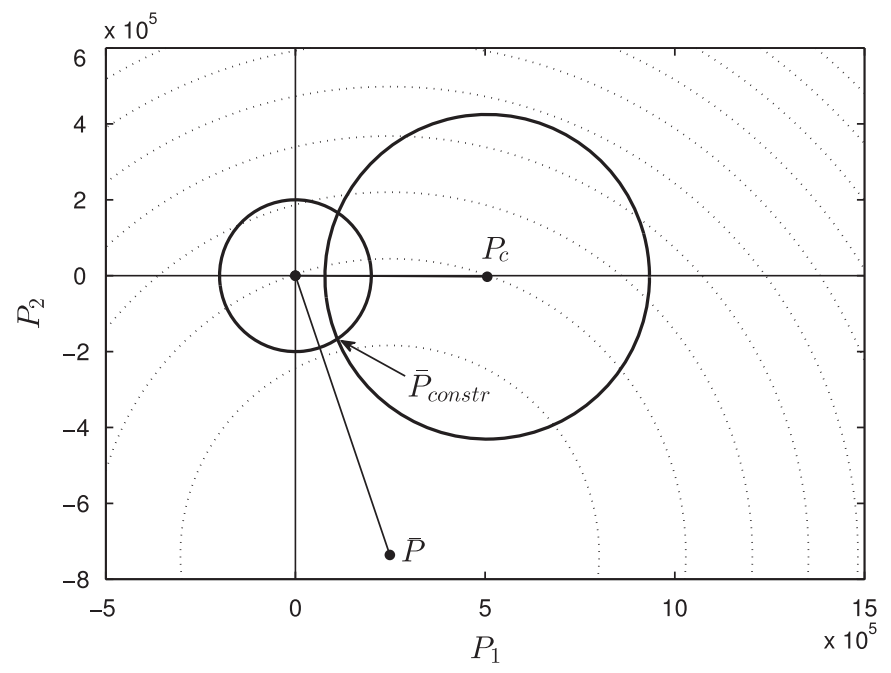

Fig. 5. Single body device in a monochromatic sea. 


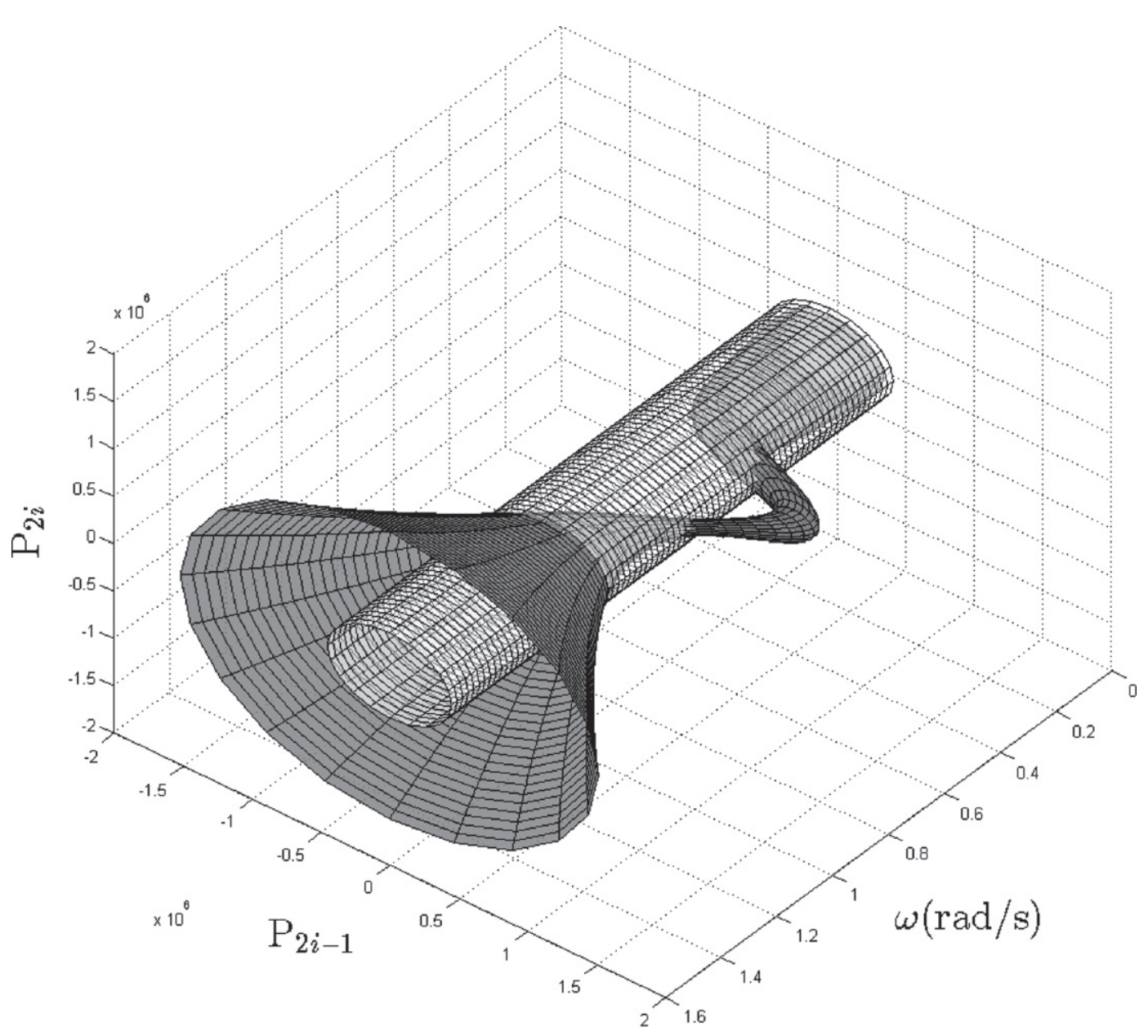

Fig. 6. Single body device with Bretschneider spectrum.

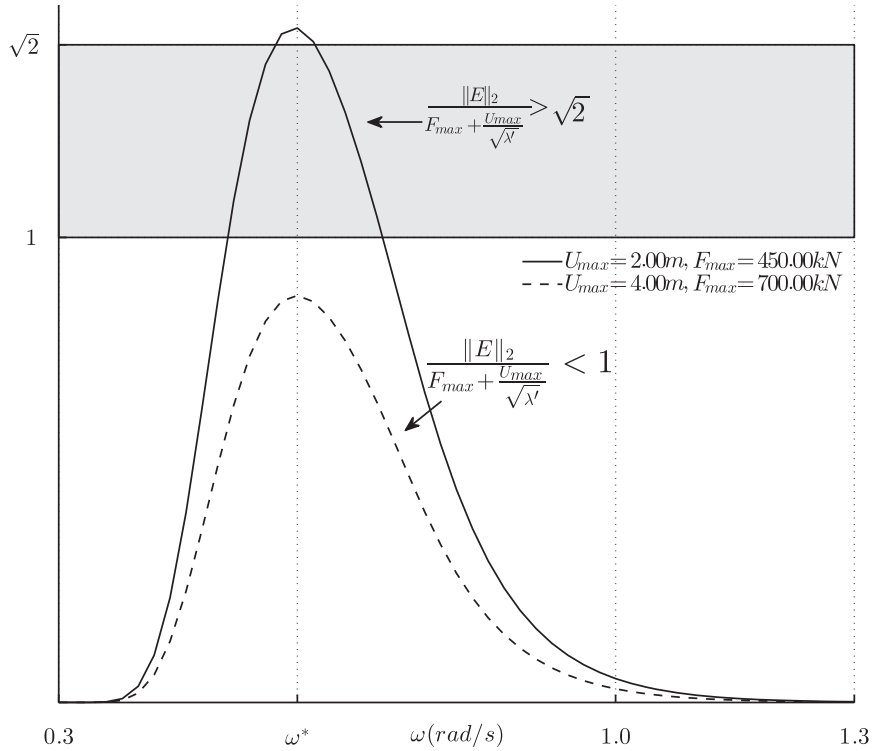

Fig. 7. Single body device with Bretschneider spectrum, using 2D projection.

For this polychromatic case, we examine two sets of constraints:

1. $\Delta U_{\max }=2 \mathrm{~m}, F_{\max }=450 \mathrm{kN}$

2. $\Delta U_{\max }=4 \mathrm{~m}, F_{\max }=700 \mathrm{kN}$
From Fig. 7, case (a) above clearly violates the constraints, while case (b) clearly (conservatively) satisfies the constraints. Further, detailed, examination can now proceed by focussing on the single frequency $\omega^{*}$ and using a plot similar to that of Fig. 5, thereby improving the insight into individual constraint satisfaction and manipulation, with the energy landscape as a background, available.

\subsection{Two-body case}

Our two-body example consists of a device as shown in Fig. 2, with the following dimensions:

- Torus inner diameter of $10 \mathrm{~m}$.

- Torus outer diameter of $20 \mathrm{~m}$.

- Torus draught of $8 \mathrm{~m}$.

- Torus mass of $1.93 \times 10^{6} \mathrm{~kg}$.

- Cylinder diameter of $8 \mathrm{~m}$.

- Cylinder draught of $30 \mathrm{~m}$.

- Cylinder mass of $1.55 \times 10^{6} \mathrm{~kg}$.

The two-body case differs only from the single body case in that the amplitude and force constraints are relative (between the two bodies) rather than absolute and, while the general form of Fig. 1 is followed, $f_{3}\left(F_{e}\right)$ is not simply the distance between circles (or, in the polyspectral case, cylinders). Fig. 8 shows the situation for 4 different sets of constraints, where constraint satisfaction is only guaranteed for $\Delta U_{\max }=0.1 \mathrm{~m}, F_{\max }=0.45 \mathrm{kN}$. 


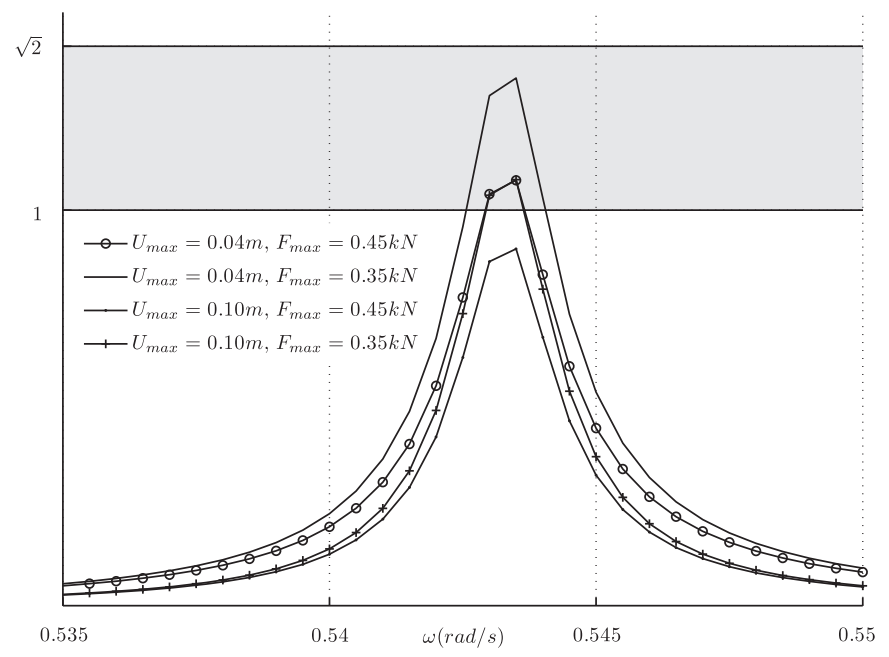

Fig. 8. Two-body device with Bretschneider sea spectrum.

Again, a more detailed examination can be obtained by resorting to the $P_{2 i} V_{s} P_{2 i-1}$ plane, with a focus on the peak frequency.

A characteristic difference between Figs. 7 and 8 is that the bandwith of the two-body system is significantly narrower, reflecting the significant difference in dynamics for the single- and two-body cases, for the range of parameters considered. The discretization effect manifested in Fig. 8, resulting in a slightly non-smooth spectral shape, is the result of numerical effects in WAMIT, rather than the frequency points chosen for the Fourier series representation.

\section{Conclusions}

In this paper, we have developed a procedure to examine if physical constraints will be satisfied for a particular wave energy device, under a particular set of sea conditions. The method does not require any simulation and does not depend on the control method used to calculate the PTO force. However, the method does require hydrodynamic model parameters which are used to evaluate the matrix $G$ in (14).

The method relies on a discretization of the equation of motion in frequency, using Fourier series, leading to a system of linear equations. A consequence of this discretization, for the polychromatic case, is the need to examine the constraint satisfaction at each discrete frequency. However, this examination can be done in a broad way first and then localized to the key (offending) frequency, leading to the use of parametric plane described by the couple $P_{2 i}, P_{2 i-1}$ (corresponding to a single frequency) for further investigation. If desired, the $P_{2 i}, P_{2 i-1}$ plane can be employed as a design tool, showing constraint features, the energy landscape and the location of the optimum PTO force. The superposition of these features allows the designer to make informed decisions regarding the value of relaxing (or, indeed, tightening) the constraints, in relation to energy capture. In particular, we envisage this tool being used in the techno-economical optimization of WECs, because it enables to show the relation between dimensions of the device (maximum PTO force and oscillation amplitude), which are related to the cost, and the amount of energy produced which is related to the revenue generated by the WEC.

The theoretical development in this paper supports any device for which a hydrodynamic model can be obtained. The examples given show one (relative) degree of motion i.e. a single body, or two-body WEC. While the method can be extended to multiple (relative) degrees of freedom, the intuitive feel for the geometric interpretation is likely to be diminished, though absolute confirmation of constraint satisfaction can still be established.

The transformation of the constraints from an infinity-norm representation to the more amenable 2-norm introduces some conservatism. However, given the development of sets of conditions guaranteeing both satisfaction and non-satisfaction of the infinity norm (based on 2-norm calculations), the margin for error is relatively small, amounting to $\pm 16 \%$ overall.

Finally, the development in this paper is based on a linear hydrodynamic WEC model. While it is known that such linear approximations can suffer a lack of fidelity for large WEC motions (due to viscous, and other, effects), there is general agreement that linear hydrodynamic models reasonably approximate device motion over the power production region of operation (Bjarte-Larsson and Falnes, 2006; Weller et al., 2013). The interplay between force constraints, amplitude constraints and optimal power capture in the power production region is the focus of this paper, while constraint analysis in survival modes, where device dynamics are likely to be significantly different due to PTO locking or unloading, would need a different (and likely nonlinear) approach. Typically, survival modes are analyzed using computational fluid dynamics (CFD) models.

\section{Appendix A}

The 2-norm $\|f\|_{2}$ of the function $f(t)$ is defined as

$\|f\|_{2}=\left(\int_{0}^{T}|f(t)|^{2} d t\right)^{1 / 2}$,

while the infinity-norm $\|f\|_{\infty}$ is defined as

$\|f\|_{\infty}=\sup _{t \in[0, T]}|f(t)|$.

A general property relating the 2-norm and the infinity-norm of a function $f$, for which the norm defined by Eq. (A.2) exists, is Atkinson and Han (2005)

$\|f\|_{2} \leq \sqrt{T}\|f\|_{\infty}$.

For a zero mean Fourier series with $N$ frequency components the inequality (Timan, 1994)

$\left\|f_{N}\right\|_{\infty} \leq \sqrt{\frac{2 N}{T}}\left\|f_{N}\right\|_{2}$

provides an upper bound for the infinity-norm as a function of the 2-norm.

\section{References}

Atkinson, K., Han, W., 2005. Theoretical Numerical Analysis: A Functional Analysis Framework. Springer.

Bacelli, G., Ringwood, J.V., Gilloteaux, J.-C., 2011. A control system for a self-reacting point absorber wave energy converter subject to constraints. In: IFAC World Congress, pp. 11387-11392.

Bjarte-Larsson, T., Falnes, J., 2006. Laboratory experiment on heaving body with hydraulic power take-off and latching control. Ocean Eng. 33 (May (7)), $847-877$.

Boyd, J.P., 2001. Chebyshev and Fourier Spectral Methods. Dover.

Cretel, J., Lightbody, G., Thomas, G., Lewis, A., 2011. Maximisation of energy capture by a wave-energy point absorber using model predictive control. In: Proceedings of the IFAC World Congress. pp. 3714-3721.

Evans, D., 1981. Maximum wave-power absorbtion under motion constraints. Appl. Ocean Res. 3 (4), 200-203.

Falnes, J., 1999. Wave-energy conversion through relative motion between two single-mode oscillating bodies. J. Offshore Mech. Arct. Eng. 121 (1), 32-38.

Falnes, J., 2000. Maximum wave-energy absorbtion by oscillating systems consisting of bodies and water columns with restricted or unrestricted amplitudes. In: Proceedings of the International Offshore and Polar Engineering Conference. pp. 420-425.

Falnes, J., 2002. Ocean Waves and Oscillating Systems: Linear Interactions Including Wave-energy Extraction. Cambridge University Press. 
Hals, J., Falnes, J., Moan, T., 2011. Constrained optimal control of a heaving buoy wave-energy converter. J. Offshore Mech. Arct. Eng. 133 (1), 011401-011415.

Ogilvie, T., 1964. Recent progress toward the understanding and prediction of ship motions. In: Proceedings of the Fifth Symposium on Naval Hydrodynamics, Bergen, Norway.

Pizer, D., 1993. Maximum wave-power absorbtion of point absorbers under motion constraints. Appl. Ocean Res. 15 (4), 227-234.
Timan, A.F., 1994. Theory of Approximation of Functions of a Real Variable. Courier Dover Publications.

Weller, S., Stallard, T., Stansby, P., 2013. Experimental measurements of the complex motion of a suspended axisymmetric floating body in regular and near-focused waves. Appl. Ocean Res. 39 (0), 137-145. 